\title{
L'ombre portée sur l'avenir de la trame verte et bleue. Quelques réflexions juridiques
}

The questionning on the futur of the green and blue weft: Some legal considerations

Marie-Pierre Camproux-Duffrène et Marthe Lucas

\section{OpenEdition}

\section{Journals}

Édition électronique

URL : http://journals.openedition.org/developpementdurable/9256

DOI : 10.4000/developpementdurable.9256

ISSN : 1772-9971

Éditeur

Association DD\&T

\section{Référence électronique}

Marie-Pierre Camproux-Duffrène et Marthe Lucas, « L'ombre portée sur l'avenir de la trame verte et bleue. Quelques réflexions juridiques », Développement durable et territoires [En ligne], Vol. 3, n

2 | Juillet 2012, mis en ligne le 12 juillet 2012, consulté le 30 avril 2019. URL : http://

journals.openedition.org/developpementdurable/9256; DOI : 10.4000/developpementdurable.9256

Ce document a été généré automatiquement le 30 avril 2019

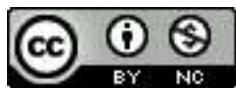

Développement Durable et Territoires est mis à disposition selon les termes de la licence Creative Commons Attribution - Pas d'Utilisation Commerciale 4.0 International. 


\section{L'ombre portée sur l'avenir de la trame verte et bleue. Quelques réflexions juridiques}

The questionning on the futur of the green and blue weft: Some legal considerations

Marie-Pierre Camproux-Duffrène et Marthe Lucas

1 La trame verte et bleue (TVB) présente de multiples intérêts sur le plan écologique, que ce soit en termes de circulation des espèces, de reconnaissance d'espaces dédiés à la biodiversité (protégée ou non protégée), d'amélioration de certaines fonctionnalités des écosystèmes, (Vimal et al., 2011) ${ }^{1}$. Juridiquement, elle constitue l'un des apports incontestables des lois Grenelle en matière de biodiversité ${ }^{2}$. Composée en partie d'espaces protégés par le droit de l'environnement et par des territoires assurant leur connexion, la trame verte et bleue concerne le milieu terrestre comme le milieu aquatique. Ce n'est pas tant le concept de maillage écologique déjà intégré au droit notamment par le réseau Natura $2000^{3}$ que celui de corridors écologiques qui est novateur en droit français (Bonnin, 2008) ${ }^{4}$. La trame se compose, en effet, à la fois de réservoirs de biodiversité et de corridors écologiques. Les premiers correspondent à de nombreux espaces bénéficiant déjà d'une protection juridique forte ou étant importants pour la préservation de la biodiversité. Quant aux corridors écologiques, ils sont " constitués des espaces naturels ou semi-naturels ainsi que des formations végétales linéaires ou ponctuelles $»^{6}$. La loi ne donne aucune indication particulière ${ }^{7}$, si ce n'est qu'ils doivent relier les réservoirs de biodiversité identifiés. Leur contenu étant librement défini, il pourra s'agir, entre autres, de nature ordinaire (forêts domaniales, etc.), d'espaces protégés méconnus en tant que réservoirs de biodiversité à savoir les espaces protégés au titre du code de l'urbanisme ${ }^{8}$ ou les espaces naturels sensibles. Représentant tout l'apport de la TVB, les corridors écologiques restent à construire selon une analyse au cas par cas. La trame se présente donc comme un outil de l'État parmi d'autres en vue de "stopper la perte de biodiversité sauvage et domestique, de restaurer et de maintenir ses capacités d'évolution »". La TVB n'étant 
pas affectée à une espèce ou un espace remarquable, toutes les collectivités sont à même de se saisir de cet outil au profit de tous types d'écosystèmes. Les acteurs locaux disposent ici d'une marge de manœuvre importante pour en délimiter le tracé.

Née des consultations du Grenelle de l'environnement, la mise en œuvre de la trame verte bleue fait également écho à l'engagement $n^{\circ} 76 \mathrm{du}$ Grenelle « Restaurer la nature en ville et ses fonctions multiples ", engagement ayant été inscrit dans la loi du 3 août $2009^{10}$. Les enjeux de la trame en milieu urbain diffèrent quelques peu de sa mise en place dans les territoires ruraux. Ainsi, le volet écologique prend-il des proportions plus importantes au regard de la densité des habitats. La trame devient une passerelle assurant la connexion entre la nature intra muros et la nature aux abords de la ville. De même, les effets de la TVB en matière d'amélioration de la qualité et de diversité des paysages ${ }^{11}$ sont particulièrement appréciés des populations urbaines qui y voient un moyen d'embellir leur cadre de vie.

La mise en place de la TVB signifie une traçabilité pérenne de corridors écologiques dans un contexte de pression foncière constante au détriment de la préservation des espaces naturels face aux besoins croissant de surface pour les activités humaines agricoles, industrielles, l'habitat. Parmi les solutions avancées pour mettre en œuvre la trame, l'idée de coupler compensation écologique et trame verte et bleue a émergé récemment (Lucas, 2011). La compensation écologique renvoie à l'obligation faite aux maîtres d'ouvrage de compenser les dommages environnementaux de leur projet subsistant après les mesures de suppression et de réduction (Steichen, 2009 ; Lucas, 2009) ${ }^{12}$. De nature écologique, elle implique de recréer ou d'améliorer un milieu naturel équivalent - sans jamais pouvoir être identique - à celui dégradé ou détruit. Se situant dans une dynamique de maintien de la diversité biologique comparable à celle de la TVB, la compensation nécessite une conservation des milieux ainsi restaurés sur une durée égale à la celle du dommage causé par l'ouvrage ou l'activité. La finalité de cet instrument coïncide ainsi avec celle de la trame verte et bleue en ce qu'elle vise à lutter contre la perte de biodiversité. Ayant lieu sur un espace ayant vocation à rester naturel, la compensation devient a priori une opportunité foncière pour la réalisation de la trame.

L'intérêt écologique du dispositif TVB ainsi que la mise en place progressive de la trame selon la mobilisation des élus ne doivent pas occulter les difficultés pratiques et juridiques de son application. Un certain flou juridique entoure en effet la mise en place de la trame verte (Camproux-Duffrène et Lucas, 2011). Dépendant de la motivation des politiques, l'avenir de la trame verte pourrait être compromis en ville par les pressions immobilières croissantes, et, ce, en raison des effets juridiques actuellement limités de la trame (1). A ce risque vient s'ajouter celui de l'utilisation de la trame à des fins de « réserves foncières locales " destinées à compenser les impacts d'aménagements endommageant l'environnement. La réunion de ces deux instruments juridiques, loin d'être anodin, n'est pas sans danger pour l'avenir de la trame et plus généralement de la diversité biologique (2).

\section{Les effets juridiques locaux limités de la TVB}

5 La faiblesse actuelle du dispositif de la trame verte et bleue s'explique non seulement par l'imprécision des moyens visant à matérialiser la trame (1.1) mais aussi et surtout par le caractère inopposable de la trame vis-à-vis d'aménagements lui portant atteinte (1.2). 


\subsection{L'imprécision des moyens juridiques visant à matérialiser la trame}

6 De façon à organiser la mise en place de la trame, le code de l'environnement prévoit l'élaboration de deux documents-cadres. Le premier se situe à l'échelon national ("Orientations nationales pour la préservation et la remise en bon état des continuités écologiques »), tandis que le second (« Schéma régional de cohérence écologique », SRCE) ${ }^{13}$ doit être décliné au niveau régional tout en prenant en compte les dispositions du premier. L'ambition de ces deux documents reste très théorique. Elle se résume au niveau national à faire un état des lieux des enjeux nationaux et frontaliers en vue de la préservation et de la remise en bon état des continuités écologiques et à définir des critères méthodologiques ${ }^{14}$, et pour le SRCE, à recenser les réservoirs de biodiversité régionaux (c'est-à-dire les espaces protégés de la trame ${ }^{15}$ ) ainsi qu'à établir la cartographie de la TVB. Les textes n'abordent ni la question de la mise en œuvre locale de la TVB ni celle de l'articulation entre le schéma régional de cohérence écologique et les outils juridiques dont disposent les collectivités locales. Or, même si les textes désignent la région comme l'unique entité chargée d'élaborer la trame, cette dernière aura besoin de l'appui et de la collaboration des autres collectivités territoriales pour matérialiser son tracé et son contenu.

7 Les lois Grenelle éludent ainsi la question des moyens concrets de réaliser la trame en pratique, si bien que les collectivités locales ne disposent pas de fondement juridique particulier pour appuyer leur création de trame verte. Concrètement, l'élaboration d'une TVB n'a pas vocation à changer le droit applicable sur les espaces déjà protégés. En ce qui concerne les corridors écologiques, composés de nature ordinaire, il ne s'agit pas d'interdire toutes activités présentes, notamment les activités agricoles. La trame vise plutôt à encourager les acteurs locaux, propriétaires fonciers, collectivités, agriculteurs, à participer à la préservation, à la gestion et, le cas échéant, à la remise en bon état des milieux. Toutefois, la question de l'incitation de ces acteurs à conserver ou à adopter une pratique conciliable avec la préservation de la biodiversité reste ouverte. Deux options sont envisageables (Durousseau, 2010) : l'affectation par le code de l'urbanisme des zones compatibles avec la trame ou la conclusion de conventions avec les propriétaires ou avec les locataires des parcelles de la trame.

8 Allant dans le sens de la première proposition, les documents d'urbanisme ont désormais comme objectif, parmi d'autres, la préservation et la création de continuités écologiques ${ }^{16}$ . Cet objet rend donc possible l'utilisation de plusieurs dispositions au bénéfice de la construction de la trame. A titre d'illustration, le schéma de cohérence territoriale (SCOT) ainsi que le plan local d'urbanisme (PLU) permettent de délimiter les espaces à protéger ${ }^{17}$. Ce mode de création de la trame lui confère une certaine assise juridique dans la mesure où la révision ou la modification de ces documents nécessite une procédure astreignante. Il serait sans doute intéressant de l'employer dans les milieux fortement urbanisés où l'affectation environnementale ou naturelle du site permet de faire face à la forte pression d'artificialisation du milieu. En revanche, les dispositions du code de l'urbanisme n'autorisent pas les collectivités à imposer des obligations de faire, c'est-à-dire à exiger des personnes privées propriétaires ou locataires du terrain un mode de gestion précis.

9 La seconde piste, celle de la contractualisation, offre davantage de souplesse quant au contenu des obligations fixées pour la gestion ou l'entretien de la trame. C'est d'ailleurs le 
mode retenu par le SRCE, lequel doit mentionner les mesures contractuelles adéquates pour atteindre les objectifs fixés par le schéma régional ainsi que les mesures d'accompagnement de la mise en œuvre des continuités écologiques pour les communes concernées par le projet de schéma ${ }^{18}$. En fonction des acteurs intéressés ou de la nature des milieux, la région pourrait choisir, par exemple de contractualiser avec les propriétaires moyennant, le cas échéant, une rémunération pour changement de pratique. Étant propriétaire d'espaces naturels, elle pourrait également conclure des baux environnementaux avec des agriculteurs. Les collectivités locales peuvent ainsi se lancer dans une politique de contractualisation afin d'assurer la mise en place de la trame à leur échelon. Cette façon de procéder rencontre toutefois deux écueils inévitables : l'obtention de l'accord des tiers et un coût élevé de mise en œuvre.

À cette imprécision sur la mise en place de la trame verte s'ajoute l'inopposabilité de celle-ci.

\subsection{Le caractère inopposable de la trame vis-à-vis d'aménagements lui portant atteinte}

11 La question du caractère inopposable de la trame revient à s'interroger sur sa portée juridique. Quelle est sur le plan juridique la force contraignante de ce dispositif vis-à-vis d'autres projets? Cette question, redondante notamment en droit de l'urbanisme (Jacquot, 2009) et plus généralement en droit de l'environnement (Maudhuit, 1997), implique d'examiner la place des normes définissant la trame, à savoir les orientations nationales et le SRCE, dans l'ordonnancement juridique actuel. Trois types de rapport juridique sont possibles: la conformité, la compatibilité et la prise en compte. La conformité interdit toute différenciation entre norme supérieure et norme inférieure, sans exiger une identité absolue entre les deux normes ${ }^{19}$. Aucun rapport de conformité n'a été prévu en matière de trame verte et bleue jusqu'à présent. La notion de compatibilité, quant à elle, implique une absence de contrariété par les normes d'application avec les options fondamentales des normes supérieures ${ }^{20}$. Selon cette définition, des atteintes mineures à la trame seraient tolérées tout en interdisant les dommages graves à la TVB nationale. Cette notion assure toutefois une meilleure pérennité à la trame que celle de prise en compte. Cette dernière, critiquée par certains juristes (Cans, 2011; Janin, 2010), signifie seulement que les dispositions de la norme supérieure ne doivent pas être ignorées. Des aménagements à la trame pourraient être prévus.

12 Légalement, les textes relatifs à la TVB prévoient que les collectivités territoriales doivent prendre en compte le SRCE ${ }^{21}$. Conformément à la définition ci-dessus, la notion de prise en compte ne saurait interdire à un document d'urbanisme de remettre en cause la cartographie de la TVB du schéma régional en décidant par exemple que la trame doit adopter un tracé différent. Or, cette hypothèse est loin d'être théorique. S'ils sont tenus de préserver les équilibres et la qualité des écosystèmes ${ }^{22}$, les documents d'urbanisme doivent de surcroit assurer des conditions d'habitat, d'emploi, de services et de transports aux populations résidantes et futures. La concrétisation de ces dernières missions peut aboutir, à terme, à remettre en cause le tracé de la trame verte, faute de pouvoir trouver d'autres disponibilités foncières. Cette faculté, pour un document d'urbanisme, de modifier en partie le tracé de la trame remet en cause sa pérennité juridique. Certes, en cas d'atteintes à la trame, les documents d'urbanisme devront 
prévoir les moyens de les compenser au titre de leur évaluation environnementale ${ }^{23}$. Conformément à la transposition de la directive $2001 / 42 / \mathrm{CE}^{24}$, les documents d'urbanisme listés à l'article L. 121-10 du code de l'urbanisme, entre autres, les SCOT, sont désormais tenus de présenter, dans la mesure du possible, les mesures destinées à compenser les incidences négatives notables que peut avoir le document sur l'environnement. Un document autorisant une zone d'urbanisation sur un espace faisant jusqu'alors office de trame verte et bleue devrait donc prévoir des moyens de compensation. Relevons toutefois le statut indéterminé de la notion de compensation à l'échelle des documents de planification, notamment quant à la forme qu'elle doit prendre et la personne responsable de leur réalisation concrète. Il y a fort à penser que cette compensation ne soit pas une compensation en termes écologiques mais une " compensation territoriale » (Gobert, 2008).

13 Par ailleurs, la loi précise que les projets publics nationaux, dont les grandes infrastructures linéaires de l'État, doivent être compatibles avec les objectifs nationaux ${ }^{25}$. Il s'agit en pratique des autoroutes, voies ferrées, gazoducs, etc., à savoir les projets fragmentant profondément les milieux naturels et de ce fait, responsables de nombreuses ruptures de continuités écologiques. Eu égard à la définition de la notion de compatibilité et à l'imprécision de la TVB nationale, la preuve d'une atteinte de manière significative à la TVB nationale par l'un de ses projets risque d'être complexe à apporter. Pour leur part, les projets publics locaux, à savoir les projets de l'État, des régions, des conseils généraux, des communautés de communes et des communes, sont tenus de prendre en compte le $\mathrm{SRCE}^{26}$. La notion de " prise en compte » accentue la probabilité que les projets s'écartent des dispositions prévues par la trame régionale. Ici aussi, les détériorations de la trame régionale sont tolérées tant qu'elles ne la détruisent pas de manière importante.

En revanche, les projets privés ne sont pas visés par la loi Grenelle II. Ils ne sont donc pas soumis à un rapport direct de compatibilité ou de prise en compte du schéma régional, à savoir la cartographie de la trame et les mesures contractuelles pour la conserver ou l'améliorer établies par le $\mathrm{SRCE}^{27}$. De façon indirecte, ces projets privés devront prendre acte de l'existence de la trame verte. D'une part, l'implantation des ouvrages se faisant conformément au contenu des documents d'urbanisme, elle ne pourra avoir lieu si ces derniers assurent une protection juridique des parcelles destinées à la TVB. D'autre part, le maitre d'ouvrage d'un projet privé est confronté à la prise en compte de la trame verte par le prisme de la réglementation des études d'impact. Conformément aux prescriptions relatives à celle-ci ${ }^{28}$, il doit donc décrire l'état initial de l'environnement, analyser les effets négatifs de son projet sur l'environnement et proposer des mesures afin d'éviter ces effets, de les réduire et, si possible, de les compenser. Si elle existe, la TVB doit être recensée dans l'état initial et si jamais le projet lui porte atteinte, le maitre d'ouvrage est tenu de proposer des mesures pour compenser la rupture des continuités écologiques.

Il ressort de cette analyse que la pérennité de la trame n'est pas garantie sur le long terme. Inopposable aux projets publics comme aux projets privés ${ }^{29}$, la trame peut encore se trouver affectée par la modification des documents d'urbanisme. Certes, dans tous ces cas de figure, le législateur prévoit une obligation de compensation des atteintes (Camproux-Duffrène et Lucas, 2011). Cependant au vu des incertitudes scientifiques que pose la compensation en nature d'une rupture définitive de continuité écologique, il eût mieux valu que le législateur instaure une protection juridique à la TVB et empêche que des projets ou des documents de planification puissent y porter atteinte. Il aurait pu par exemple imposer que les documents d'urbanisme et les projets nationaux et locaux aient 
à respecter la délimitation de la TVB régionale, sauf à démontrer l'existence d'un intérêt public majeur.

Ainsi, la concrétisation de la trame verte repose essentiellement sur l'engagement des acteurs locaux. Cependant, à l'heure actuelle, cet outil ne bénéficie pas d'une protection juridique forte. Il en résulte qu'avant même sa mise en place, la TVB n'est pas à l'abri d'un grignotage progressif puisqu'un projet national ou territorial peut y porter atteinte dans une certaine mesure (Janin, 2010) ${ }^{30}$. Le manque d'opposabilité de son tracé au regard des documents d'urbanisme et des grands projets dans chaque région fait ainsi douter des priorités écologiques en matière d'aménagement du territoire. Les effets juridiques de la trame verte méritent donc d'être renforcés de façon à ne pas ruiner les efforts entrepris localement et les objectifs poursuivis.

Outre cette critique et le manque de moyens juridiques, la mise en place de la TVB se trouve ralentie par le manque de moyens financiers alloués à cette politique. Au vu des difficultés de mise en œuvre, les collectivités peuvent être séduites par l'approche consistant à voir dans la trame verte une "réserve foncière locale " destinée à la compensation écologique d'aménagements impactant l'environnement.

\section{La trame verte et bleue: une « réserve foncière locale » à disposition pour compenser des aménagements impactant l'environnement?}

18 La trame verte et bleue ne doit pas être une opportunité pour enfermer la nature, délimiter l'espace consacré dans chaque partie du territoire. En d'autres termes, elle ne doit pas aboutir à la création d'autoroutes pour la faune, la flore (verte) et l'eau (bleue) avec des aires de repos et de ravitaillement. La biodiversité existe aussi en ville ou sur les terres agricoles. Pourtant, la tentation est grande de voir dans la TVB un outil demeurant à disposition en tant que réserve foncière locale pouvant servir à compenser localement des impacts écologiques de projets d'aménagement ou d'activité (Lucas, 2011). Cette proposition répondrait par ailleurs à un réel souci des maîtres d'ouvrage qui, devant compenser les dommages environnementaux résiduels de leur projet, peinent à trouver des terrains disponibles permettant une compensation écologique via la création ou la restauration d'un milieu naturel. Ils font de ce fait très souvent appel aux collectivités territoriales pour bénéficier d'un appui dans leur recherche de foncier. Conjuguer la mise en place et la gestion d'une trame locale avec la réalisation de leurs mesures compensatoires pourrait faciliter leur démarche et augmenter ainsi leurs chances d'obtenir l'autorisation administrative requise, voire dans certains cas empêcher un refus d'autorisation. Certains acteurs locaux pourraient ainsi concevoir la trame comme un réservoir de nature permettant la justification de la dégradation de milieux naturels environnants. D'autant que le raisonnement pourrait être tenu également en ce qui concerne les mesures de réparation en cas de dommages environnementaux. Quelques collectivités dont la Communauté Urbaine de Strasbourg (CUS) ont d'ailleurs déjà envisagé cette articulation ${ }^{31}$.

19 Se servir de la TVB pour assurer la compensation ou la réparation des atteintes à l'environnement suscite d'autant plus l'intérêt des acteurs locaux qu'il existe des craintes quant au financement de la trame. Comme précédemment souligné, la loi Grenelle II n'assure pas de source de financement à la trame. Celle-ci devra être déterminée au cas 
par cas par les régions ou les autres collectivités territoriales participant à sa mise en place. Il n'est d'ailleurs pas prévu de décret d'application sur ce point bien que les communes aient demandé des compensations financières qu'elles n'ont pas obtenues (Roux-Goeken, 2009) ${ }^{32}$. L'unique ressource envisagée par la législation actuelle semble être celle issue de la taxe départementale des espaces naturels sensibles ${ }^{33}$.

Face à ce constat, la participation financière d'acteurs privés à la création de la trame au titre de compensations écologiques parait séduisante puisqu'elle aboutit à un double résultat. Filiale de la Caisse des Dépôts des Consignations (CDC), la CDC Biodiversité se situe à la fois sur les rangs pour assurer dans certaines régions l'élaboration de la TVB et pour prêter main forte aux aménageurs publics comme privés devant ou souhaitant compenser leurs impacts (CDC Biodiversité, 2008). En ce sens, elle a d'ailleurs déjà conclu avec la ville de Paris une étude de faisabilité de la mise en place d'un mécanisme de compensation «visant à renforcer le rôle de corridor biologique joué par la Petite Ceinture, élément majeur de la trame verte parisienne » (Fabrégat, 2011).

21 Selon l'« approche par l'offre ", la CDC Biodiversité anticipe le besoin de compensation sur un territoire et réalise en amont des opérations visant à restaurer certains milieux sans connaître au préalable les impacts des projets correspondant (CDC Biodiversité, $2008)^{34}$. Ce n'est que postérieurement à ces opérations d'ingénierie écologique, lorsqu'elle est saisie d'une demande précise de compensation que la CDC propose de vendre les services réalisés sur le terrain. À titre d'illustration, elle a déjà fait l'acquisition d'un espace dans la plaine de Crau et a lancé, avec le soutien du ministère en charge de l'écologie, la première "réserve d'actifs naturels » sur cet écosystème unique, le 10 août $2010^{35}$. Cette expérimentation a donné lieu à la signature de deux conventions avec le ministère encadrant la création une réserve d'actifs naturels permettant de compenser les impacts négatifs à la biodiversité ${ }^{36}$. Il s'agit pour CDC Biodiversité de restaurer des terrains dénaturés et de les vendre à ceux qui portent atteinte à la biodiversité à titre de compensation. En pratique, l'opération menée sur la commune de Saint Martin de Crau dans les Bouches-du-Rhône a consisté à restaurer l'habitat d'espèces rares et menacées en transformant plus de 300 hectares d'un verger industriel en une steppe pâturée par les moutons. Au terme de trois ans, l'expérimentation devrait comprendre quatre sites dans des contextes écologiques différents. La CDC Biodiversité a vendu fin 2011 ces premières unités de biodiversité (Camproux-Duffrène, 2009 ; Laramée de Tannenberg, 2011).

En raisonnant sur ce modèle, certains acteurs disposent d'une opportunité économique considérable en investissant dans la construction de la trame verte. Réalisée à toutes les échelles et dans tous les départements, la trame constitue une source de foncier considérable à transformer en "réserve d'actifs locale» revendus ensuite à des aménageurs devant compenser leurs impacts. Cette pratique aurait pour conséquence de faire de la TVB une réserve foncière locale, un «stock de biodiversité » (Sinaï, 2011) mis à la disposition des maîtres d'ouvrage. Cela signifie admettre une certaine conception de la nature selon laquelle des unités de biodiversité, ou unités d'actifs naturels, dématérialisées, créées par certains opérateurs peuvent s'échanger contre de l'argent. Or, de l'évaluable en argent à la marchandisation, la frontière est mince. Si ces unités sont échangeables contre de l'argent, ce qui n'est pas l'objet de la critique, la compensation étant admise par la loi, elles pourraient également devenir fongibles c'est-à-dire interchangeables. Découlant concrètement du caractère fongible des unités, un aménageur pourrait acheter des unités de biodiversité (correspondant à des actions en faveur de la préservation du Grand Hamster) pour compenser la destruction d'une zone 
humide (Camproux-Duffrène, 2009). Il est dès lors primordial que le critère de base et le seul de cette opération d'échange soit l'équivalence écologique - la proximité territoriale en faisant partie - afin d'éviter des aberrations écologiques.

Par ailleurs, selon la position actuelle défendue, la CDC-Biodiversité resterait propriétaire des terrains vendant uniquement la prestation de service réalisée (l'amélioration écologique $\mathrm{du}$ terrain). Elle s'est imposée la contrainte d'une affectation environnementale des terrains achetés pour trente ans. Cette durée, aussi longue soitelle, demeure cependant insuffisante pour compenser la destruction définitive de milieux naturels par le projet.

Appliquée en milieu urbain, cette approche offerte par les unités d'actifs naturels ou plus largement la mise en place de réserve foncière locale risque d'aboutir à une concentration de la nature dans ces seuls espaces. Or, ni la compensation qui répond plutôt à la détermination de quota pour atteindre une réelle additionnalité écologique, ni la trame verte qui ne vise pas à devenir le sanctuaire d'une biodiversité protégée ne raisonne en termes d'intensification de la nature. Rappelons, enfin, que la trame verte n'est pas à même d'assurer la pérennité de la compensation ou de la réparation écologique, dans la mesure où son tracé est révisable et où des projets d'aménagement peuvent y porter atteinte. Il y aurait donc d'un coté la destruction définitive d'un milieu, de l'autre la participation à la création d'une trame dont la viabilité est soumise aux aléas du développement du territoire.

\section{Conclusion}

Pour conclure, il convient d'appeler les acteurs à la vigilance pour que cet outil juridique, la TVB, apparemment mis au service de la protection de la nature ne devienne pas un moyen de mettre la nature en réserve ou "en conserve " (Cans, 2010). Il s'agit encore moins d'en faire un commerce local entre aménageurs et producteurs de nature en l'absence de toute équivalence écologique. Dans cette hypothèse comme de manière plus générale, la postérité de la trame repose actuellement moins sur un encadrement juridique abouti que sur les dynamiques locales et les moyens techniques et financiers mis à la disposition des acteurs. L'instrument juridique qu'est la mise en place de la trame verte et bleue n'est pas de ceux qui pourront contribuer au nouveau principe de non régression du droit de l'environnement que Michel Prieur appelle de ces vœux (Prieur, 2012).

\section{BIBLIOGRAPHIE}

Bonnin M., 2008, Les aspects juridiques des corridors biologiques, Vers un troisième temps de la conservation de la nature, Paris, L'Harmattan.

Bonnin M., 2010, « Les corridors écologiques dans le droit français », Les Communautés et la trame verte et bleue, Synthèse, ACUF, p. 37-39. 
Camproux-Duffrène M.-P., 2009, « La création d'un marché d'unité de biodiversité est-elle possible? ", RJE, $\mathrm{n}^{\circ} 1, \mathrm{p} .69-79$.

Camproux-Duffrène M.-P., Lucas M., 2011, « La trame verte et bleue : un flou juridique à apprécier localement », Revue d'Allemagne et des Pays de langue allemande, tome 43, n4-2011, p. 519-530.

Cans C., 2010, « Aires protégées et connectivité écologique », journée d'étude « La loi Grenelle 2 et la connectivité écologique. Problèmes juridiques posés par la Trame verte et la Trame bleue », Université Jean Moulin - Lyon 3, 18 novembre 2010.

Cans C., 2011, « La protection de la nature dans la loi portant engagement national pour l'environnement, ou comment picorer dans une loi pour reconstruire une génétique des normes ", RJE, numéro spécial, p. 95-114.

CDC Biodiversité, 2008, « Un nouvel acteur en France pour la biodiversité », dossier de presse du 19 février 2008, 20 p.

Durousseau M., 2010, « "La boîte à outils” : conventions - servitudes - compensation - évaluation environnementale - acquisitions foncières... », journée d'étude « La loi Grenelle 2 et la connectivité écologique. Problèmes juridiques posés par la Trame verte et la Trame bleue », Université Jean Moulin - Lyon 3, 18 novembre 2010.

Fabrégat S., 2011, « Paris adopte son plan biodiversité », actu-environnement, 15 novembre 2011, http://www.actu-environnement.com/ae/news/paris-plan-biodiversite-trame-vertebleue-14113.php4, consulté le 30 novembre 2011.

Gobert J., 2008, «Compensation territoriale, justice et inégalités environnementales aux EtatsUnis », Espace Populations, Sociétés, n²1-2008, p. 71-82.

Gobert J., 2009, « Compromis compensatoires : une régulation socio-politique des conflits environnementaux en Allemagne?", Revue d'Allemagne et des Pays de langue allemande, vol. 41, p. 379-399.

Jacquot H., 2009, «Sur la règle de la compatibilité limitée en droit de l'urbanisme », in : Fatôme E., Fressoz P., Gonod P. et al. (dir.), « Terres du droit. Mélanges en l'honneur d'Yves Jégouzo », Dalloz, p. 607-618.

Janin P., 2010, « Nature et portée juridiques des documents constitutifs de la TVB », journée d'étude « La loi Grenelle 2 et la connectivité écologique. Problèmes juridiques posés par la Trame verte et la Trame bleue », Université Jean Moulin - Lyon 3, 18 novembre 2010.

Laramée de Tannenberg V., 2011, « La France invente la compensation de perte de biodiversité », Journal de l'environnement, 11 octobre 2011.

Lucas M., 2009, « La compensation environnementale, un mécanisme inefficace à améliorer », Revue Juridique de l'Environnement, $n^{\circ} 1-2009$, p. 59-68.

Lucas M., 2011, «Intérêts et limites d'une compensation écologique d'infrastructures linéaires de transport via une infrastructure verte », journées techniques nationales « Les mesures compensatoires dans les projets d'infrastructures de transport terrestre. Aspects réglementaires et retours d'expériences ", 29 et 30 mars 2011, Aix en Provence.

Maudhuit S., 1997, « La notion de compatibilité en droit de l'environnement », BDEI, n³, p. 2-10.

Planchet P., 2010, « Le droit de l'urbanisme et la connectivité écologique », journée d'étude « La loi Grenelle 2 et la connectivité écologique. Problèmes juridiques posés par la Trame verte et la Trame bleue », Université Jean Moulin - Lyon 3, 18 novembre 2010. 
Prieur M., 2012, «Le nouveau principe de «non régression » en droit de l'environnement », in : Prieur M., Sozzo G. (dir.), La non-régression en droit de l'environnement, Bruxelles, Bruylant, p. 5-46.

Roux-Goeken V., 2009, « Trame verte et bleue : les maires veulent des compensations », Journal de l'Environnement, 23 novembre 2009, http://www.journaldelenvironnement.net/article/ trame-verte-et-bleue-les-maires-veulent-des-compensations,14144, consulté le 15 novembre 2011.

Sinaï A., 2011, « Trame verte et bleue : premières expérimentations ", actu-Environnement.com, 26 janvier 2011, http://www.actu-environnement.com/ae/news/experimentations-rame-verteet-bleue-11826.php4, consulté le 15 novembre 2011.

Steichen P., 2009, « Le principe de compensation : un nouveau principe du droit de l'environnement? ", in : Cans C. (dir.), La responsabilité environnementale. Prévention, imputation, réparation, Paris, Dalloz, p. 143-162.

Vimal R., Bernard C., Letourneau A., Bernier A., Thompson J., 2011, Trame verte et bleue : quelle approche spatiale pour quel réseau écologique? Rapport réalisé au Centre d'Écologie Fonctionnelle et Évolutive (CNRS), Montpellier, 65 p., http://www.cefe.cnrs.fr/images/stories/DPTGouvernance/ dynamique-systemes-socio-ecologiques/chercheurs/ruppert_vimal/

Vimal_et_al_Rapport_2011.pdf, consulté le 22 mars 2012.

\section{NOTES}

1. La fragmentation des écosystèmes étant considérée comme l'une des causes majeures de disparition de biodiversité, rétablir ou conserver ses liaisons constitue un moyen d'assurer la dispersion et la migration des espèces, les échanges génétiques, les déplacements suite aux effets du changement climatique.

2. Loi $n^{\circ} 2009-967$ de programmation relative à la mise en œuvre du Grenelle de l'environnement du 3 août 2009 dite «loi Grenelle I " (JORF n 0179 du 5 août 2009); Loi n²010-788 portant engagement national pour l'environnement du 12 juillet 2010 dite loi Grenelle II (JORF $\mathrm{n}^{\circ} 0160 \mathrm{du}$ 13 juillet 2010).

3. Le réseau Natura 2000 se compose, à partir de 1992, de sites Natura 2000 désignés en fonction d'espèces ou d'habitats à protéger en Europe. V. Directive $n^{\circ} 79 / 409 /$ CEE du conseil du 2 avril 1979 concernant la conservation des oiseaux sauvages (JOCE $n^{\circ}$ L103 du 25 avril 1979) et directive 92/43/CEE du conseil du 21 mai 1992 concernant la conservation des habitats naturels ainsi que de la faune et de la flore sauvages (JOCE $\mathrm{n}^{\circ} \mathrm{L} 206 \mathrm{du} 22$ juillet 1992).

4. Même si le préambule de la charte de l'environnement adossée à la constitution et l'article L. 110-1 du Code de l'environnement ne distinguent pas la nature ordinaire de la nature remarquable, la protection juridique des espaces et des espèces s'est jusqu'à présent davantage focalisée sur la nature menacée.

5. Pour la trame verte, cela correspond ainsi notamment aux parcs nationaux, aux réserves naturelles, aux sites Natura 2000, aux périmètres d'un arrêté de protection de biotopes, ainsi qu'aux bandes enherbées de cinq mètres de large à partir de la rive de cours d'eau (art. L. 371-1 II C. env.). Pour la trame bleue, il s'agit des cours d'eau, partie de cours d'eau ou canaux dont le bon état écologique et le rôle de réservoir biologique ont été reconnus ; ceux permettant d'assurer le transport suffisant des sédiments et la circulation des poissons migrateurs; tout ou partie des zones humides devant être préservée ou remise en état de façon à atteindre les objectifs de qualité et de quantité des eaux fixés par la directive cadre sur l'eau (art. L. 371-1 III C. env.).

6. Article L. 371-1 II $2^{\circ} \mathrm{C}$. env.

7. Loi $n^{\circ}$ 2010-788 du 12 juillet 2010, préc. 
8. À l'exemple des zones naturelles ou forestières des plans locaux d'urbanisme. Ces zones sont ainsi classées en raison soit de la qualité des sites, des milieux et espaces naturels, des paysages et de leur intérêt, soit de l'existence d'une exploitation forestière, ou encore en raison de leur caractère d'espaces naturels (art. R. 123-8 C. urb.).

9. Article 23 de la loi $n^{\circ}$ 2009-967. Parmi les autres mesures prises dans ce but, se trouvent la création d'aires protégées terrestres sur une superficie au moins égale à $2 \%$ du territoire terrestre métropolitain, la création d'aires marines protégées couvrant $10 \%$ des eaux placées sous la souveraineté de l'État, des plans de conservation ou de restauration des espèces végétales et animales en danger critique d'extinction, des plans de lutte contre les espèces exotiques envahissantes, l'élaboration de stratégies régionales et locales de la biodiversité, etc.

10. Article 7 de la loi dite Grenelle I, préc.

11. Article L. 371-1 I $6^{\circ} \mathrm{C}$. env.

12. Article L. 122-3 C. env.

13. Article L. 371-3 C. env. À ce jour, et bien que des régions pilotes comme l'Alsace, la Picardie et Rhône Alpes soient bien avancées en terme d'élaboration de la TVB, aucun schéma régional de cohérence écologique n'a été finalisé.

14. Article L. 371-2 C. env.

15. Article L. 371-1 C. env.

16. Selon la version de l'article L. 110 du Code de l'urbanisme telle qu'issue de la loi Grenelle II, la préservation de la biodiversité, notamment par la conservation, la restauration et la création de continuités écologiques constitue un objectif à poursuivre par les collectivités publiques.

17. Respectivement articles L.122-1 C. urb. et L.123-1-7 C. urb. Serait également envisageable le classement des boisements et éléments arborés faisant partie de la trame en Espace Boisés Classé (art. L.130-1 C. urb.).

18. Article L. 371-3 C. env.

19. CE, 26 novembre 1969, Société agricole et forestière de l'Eure, $\mathrm{n}^{\circ} 72825$, ccl. M. Rougevin-Baville, AJDA, 1970, p. 239-241.

20. CE, Ass., 22 février 1974, Adam, n 91848 et 93520.

21. Article L. 371-3 C. env.

22. Article L.121-1 C. urb.

23. Article L. 121-11 C. urb.

24. Directive 2001/42/CE du Parlement européen et du Conseil du 27 juin 2001 relative à l'évaluation des incidences de certains plans et programmes sur l'environnement (JOCE $\mathrm{n}^{\circ} \mathrm{L} 197$ du 21 juillet 2001), transposée en droit français par l'ordonnance 2004-489 du 3 juin 2004 portant transposition de la directive 2001/42 du Parlement et du Conseil européen du 27 juin 2001 (JORF $\mathrm{n}$ - 129 du 5 juin 2004), le décret 2005-613 du 27 mai 2005 pris pour application de l'ordonnance 2004-489 du 3 juin 2004 (JORF $\mathrm{n}^{\circ} 124$ du 29 mai 2005) et le décret 2005-608 du 27 mai 2005 relatif à l'évaluation des incidences des documents d'urbanisme sur l'environnement et modifiant le code de l'urbanisme (JORF n 124 du 29 mai 2005).

25. Article L. 371-2 C. env.

26. Article L. 371-3 C. env.

27. Les permis de construire constituent une exception relative à cette affirmation. Délivrés conformément aux documents d'urbanisme en vigueur, lesquels sont supposés prendre en compte le SRCE, les permis de construire respecteront ainsi de façon indirecte ce dernier.

28. V. article L. 122-3 C. env.

29. Le refus d'autorisation de projets en raison de l'existence d'une trame verte et bleue n'est certes pas exclu. Seulement la réglementation actuelle n'établissant pas un rapport d'opposabilité strict permet largement des atteintes ponctuelles qui, prises individuellement, ne devraient pas remettre en cause les bénéfices de la trame, mais qui, en s'additionnant, risquent de nuire aux finalités poursuivies par cette dernière. 
30. À noter, une déclaration de projet adoptée par l'État peut procéder aux adaptations nécessaires de certains documents dont le SRCE (art. L. 300-6 al. 2 C. urb.), allant ainsi à l'encontre des objectifs régionaux de la TVB.

31. En atteste les opérations de renaturation de l'étang de Bohrie sur la commune d'Oswald. Destinées à compenser les effets de la construction du tramway à Strasbourg en 2007, elles ont abouti à une réelle connexion entre deux écosystèmes.

32. Sur ce point, depuis la rédaction de l'article en novembre 2011, un « fonds d'investissement pour la biodiversité et la restauration écologique » a été instauré auprès du ministre chargé de la protection de la nature ayant pour objet de contribuer au financement de projets et programmes favorisant notamment la préservation et la remise en état des continuités écologiques. Les ressources de ce fonds proviennent du budget de l'état. V. décret $n^{\circ}$ 2012-228 du 16 février 2012 relatif au fonds d'investissement pour la biodiversité et la restauration écologique (JORF du 18 février 2012).

33. Selon l'article L. 371-5 du code de l'environnement, "Les départements peuvent être maître d'ouvrage, ou exercer une mission d'assistance à maître d'ouvrage dans le cadre des règles de la commande publique, pour tous les travaux contribuant à la préservation ou à la remise en bon état des continuités écologiques sur la trame verte et la trame bleue d'un schéma régional de cohérence écologique adopté. Ils peuvent, pour les missions autres que celles d'assistance à maître d'ouvrage, mobiliser à cet effet le produit de la taxe départementale des espaces naturels sensibles".

34. Selon l'«approche par la demande ", la CDC Biodiversité est, dans la majorité des cas, directement sollicitée par un agent privé ou public dont la compensation à réaliser a déjà été déterminée par l'autorité administrative ayant autorisé le projet. Elle s'engage alors à lui trouver le terrain disponible, et à en assurer la gestion et la pérennité.

35. Le communiqué de presse de madame Chantal Jouanno n'a d'ailleurs pas manqué de dresser le bilan du traitement des conséquences de la rupture d'un oléoduc qui s'était produite il y a deux ans, non loin de là.

36. Présentation de la première «Réserve d'Actifs Naturels» en France sur le site de Cossure à Saint-Martin-De-Crau (Bouches-du-Rhône) par Chantal Jouanno, Secrétaire d'État chargée de l'Écologie, Augustin de Romanet, Directeur général de la Caisse des Dépôts et Laurent Piermont, Président - Directeur général de CDC Biodiversité Dossier de presse, 11 mai 2009.

\section{RÉSUMÉS}

La trame verte et bleue (TVB) constitue un instrument novateur dans l'arsenal juridique de protection de l'environnement en ce qu'il met en évidence l'importance de la connectivité de l'ensemble des écosystèmes qu'ils soient protégés ou non. L'imprécision des moyens de sa réalisation ainsi que le caractère inopposable de la trame mettent toutefois en cause l'effectivité et la pérennité de cet outil écologique. Le dispositif actuel révèle ainsi plusieurs insuffisances juridiques non négligeables à l'heure où certains acteurs proposent de créer via la TVB un espace où regrouper les mesures compensatoires prises suite à différents projets d'aménagements.

The concept of green corridors network (GCN) is an innovative legal tool because it underlines the importance of keeping connected ecosystems, protected or not. But the lack of precision on how it must be implemented and the fact that GCN are not opposable dramatically reduce the effectiveness and the durability of this tool. The current dispositive shows serious juridical lacks 
although there are ambitious proposals based on the application of the concept of green corridors network such as to mutualise ecological offsets resulting from new infrastructures implantations.

\section{INDEX}

Mots-clés : aménagement, compensation écologique, pérennité, réserve d'actifs naturels, trame verte

Keywords : durability, ecological offset, french mitigation banking, infrastructure, network of green corridors

\section{AUTEURS}

\section{MARIE-PIERRE CAMPROUX-DUFFRÈNE}

Marie-Pierre Camproux-Duffrène, maître de conférences HDR à l'Université de Strasbourg, est Directrice du Centre de Droit de l'Environnement de Strasbourg (CDES) et responsable d'un master 2 «droit de l'environnement et des risques». Ses recherches portent sur le statut juridique de l'animal, la réparation des atteintes à l'environnement et la santé, la préservation de la biodiversité via les mécanismes de droit civil.

\section{MARTHE LUCAS}

Marthe Lucas est doctorante du CDES et finit sa thèse relative à l'étude juridique de la compensation écologique. Au-delà, elle étudie les questions d'évaluation sur l'environnement et d'aménagement territorial ainsi que les outils juridiques de protection des espaces naturels. 